\title{
Interleukin 8 in middle ear fluid during acute otitis media: correlation with aetiology and bacterial eradication
}

Eugene Leibovitz, Ron Dagan, Joseph H Laver, Lolita Piglansky, Simon Raiz, Miguel R Abboud, Dan M Fliss, Alberto Leiberman, Asher Barzilai

\begin{abstract}
Objectives-To study the concentration of interleukin 8 (IL-8) in the middle ear fluid of children with acute otitis media and the association between IL-8 concentrations, aetiology of acute otitis media, and bacteriological sterilisation.

Study design-Middle ear fluid was obtained by tympanocentesis at enrolment (day 1 ) and on day $4-5$ in 81 children aged 3-36 months with acute otitis media who received antibiotic treatment. IL-8 concentrations were measured by enzyme linked immunosorbent assay.

Results-101 samples were obtained on day 1 and 47 samples on day 4-5. 94 pathogens were isolated in 79 of 101 samples obtained on day 1: 56 Haemophilus influenzae, 35 Streptococcus pneumoniae, 2 Moraxella catarrhalis, and 1 Streptococcus pyogenes. Among 40 paired, initially culture positive samples, sterilisation was achieved on day 4-5 in 22 but not in 18 (13 $\mathrm{H}$ influenzae, $2 \mathrm{~S}$ pneumoniae, and $3 \mathrm{H}$ influenzae and $S$ pneumoniae concomitantly). IL-8 was detected in 96 of 101 and 46 of 47 samples obtained on days 1 and 4-5, respectively. Mean (SD) IL-8 concentration on day 1 was significantly higher in culture positive than in negative samples (12 636 (23 317) $v 5920$ (7080) pg/ml). In paired samples, IL-8 concentration fell in 12 of 22 ears in which sterilisation was achieved and in 9 of 21 ears with persistent or new infection. Mean (SD) IL-8 concentrations on day 4-5 were significantly higher in culture positive than in negative samples (15 $420(15418) v 6695$ (5092) pg/ ml).
\end{abstract}

Conclusions-Higher IL-8 concentrations are found in culture positive middle ear fluid in acute otitis media. Bacterial eradication is associated with a fall in these concentrations.

(Arch Dis Child 2000;82:165-168)

Keywords: otitis media; interleukin 8; Haemophilus influenzae; Streptococcus pneumoniae

Acute otitis media is the most common cause of antibiotic administration in infants and children. ${ }^{12}$ Despite the many antibiotics available for its treatment, therapeutic failures, persistence of middle ear fluid effusion, and recurrences of the disease occur frequently. A better understanding of the pathogenesis and the inflammatory process associated with acute otitis media might be helpful in developing more effective strategies for the management and prevention of this disease. ${ }^{34}$

Previous findings suggest that an activated local immune system is present in the middle ear fluid of patients with chronic otitis media with effusion. ${ }^{5}$ Considerable amounts of tumour necrosis factor $\alpha$, interleukin 1 (IL-1), IL-2, and $\gamma$ interferon were found in the middle ear fluid of children with chronic otitis media with effusion; higher tumour necrosis factor $\alpha$ concentrations were found in older children and in those patients who underwent multiple tympanostomies. ${ }^{6}$ The endotoxin of Gram negative bacteria was found to be a potent amplifier of IL-8 production in middle ear fluid samples from children with chronic otitis media with effusion. ${ }^{7}$ IL-8 was found in $75-92 \%$ of samples from children and adults with chronic otitis media with effusion. ${ }^{8}{ }^{9}$ Storgaard and colleagues ${ }^{10}$ found significantly higher IL-8 concentrations in middle ear fluid from children with acute otitis media than from children with chronic otitis media with effusion, and in culture positive than in culture negative samples.

In our study, we measured the concentrations of IL-8, a cytokine that is a potent chemoattractant for polymorphonuclear leucocytes, in the middle ear fluid of children with acute otitis media, before and during antibiotic treatment. The presence of IL- 8 in the middle ear fluid of these children was then correlated with the aetiology of the disease and the efficacy of the antibiotic treatment.

\section{Patients and methods}

Our study was conducted from October 1995 to May 1997. The study group included children aged 3-36 months with acute otitis media enrolled in different studies looking at the bacteriological and clinical efficacy of different antibiotics. These studies were performed at Soroka University Medical Centre and required the performance of two tympanocenteses (at enrolment and on day 4-5 of treatment). Informed consent was obtained at enrolment from parents or guardians. The diagnosis of acute otitis media was based on symptoms of fever, irritability or earache, signs of inflammation (red or yellow colour or bulging) of the tympanic membrane, and the presence of middle ear fluid as documented by tympanocentesis. All enrolled patients had an acute illness of seven days or fewer duration; 
they had no spontaneous perforation and no tympanostomy tubes present. After enrolment, patients received different antibiotic treatments and were followed at least to day $4-5$ with a second tympanocentesis and middle ear fluid sample culture (performed only in those patients where a pathogen was isolated on day 1 and also in those patients who had negative cultures on day 1 but deteriorated during treatment).

\section{STUDY PROTOCOL}

All patients had tympanocentesis performed at enrolment by an otolaryngologist. Antisepsis of the ear canal before tympanocentesis was done with $70 \%$ alcohol instilled for one minute. After removal of the alcohol by suction, and with the use of a 20 gauge spinal needle attached to a $1.0 \mathrm{ml}$ sterilised tuberculin syringe, the anteroinferior portion of the intact tympanic membrane was punctured. The fluid $(0.1-0.2 \mathrm{ml})$ was immediately aspirated into the sterile syringe by suction and part of it applied to a sterile swab, which was sent for bacteriological culture in transport medium (MW173 Amies medium; Transwab; Medical Wire and Equipment, Potley, UK) for processing within 12 hours at the clinical microbiology laboratory. The remainder of the sample was frozen at $-70^{\circ} \mathrm{C}$ and stored for $6-12$ months before cytokine determination.

After tympanocentesis, patients were randomised to receive various antibiotic treatments (amoxycillin or cefaclor for 10 days, azithromycin or intramuscular ceftriaxone for three days).

\section{BACTERIOLOGY}

The identification methods used for all pathogens recovered have been described elsewhere. ${ }^{11}$

IL-8 ASSAY

The exact volume of the samples $(0.1-0.2 \mathrm{ml})$ was measured and cold $0.9 \%$ sodium chloride added to a precise total volume of $1 \mathrm{ml}$. Samples were then centrifuged at $4^{\circ} \mathrm{C}$ for 10 minutes to remove cellular elements. Aliquots of $0.2 \mathrm{ml}$ of the supernatant were refrozen at $-70^{\circ} \mathrm{C}$.

On the day of the IL- 8 assay, samples were diluted one in 10 using the assay diluent supplied with the kit. Samples of 50-100 $\mu \mathrm{l}$ were dispensed into 96 well microtitre plates of the "Quantikine" kit (R\&D Systems, Minneapolis, Minnesota, USA) and incubated at room temperature with $100-200 \mu$ l of anticytokine horseradish peroxidase conjugate for

Table 1 Interleukin 8 (IL-8) concentrations ( $\mathrm{pg} / \mathrm{ml}$ ) in all middle ear fluid samples obtained from children with acute otitis media, relation to aetiology of the disease and day of tympanocentesis

\begin{tabular}{lll}
\hline & Day 1 & Day 4-5 \\
\hline All samples & $11173(21033)(\mathrm{n}=101)$ & $9978(11208)(\mathrm{n}=47)$ \\
Culture positive & $12636(23317)(\mathrm{n}=79)$ & $14396(14512)(\mathrm{n}=21)$ \\
Culture negative & $5920(7080)(\mathrm{n}=22)$ & $6414(5742)(\mathrm{n}=26)$ \\
Pnc alone & $9571(9756)(\mathrm{n}=20)$ & $20850(5174)(\mathrm{n}=2)$ \\
HI alone & $15320(30966)(\mathrm{n}=41)$ & $13551(17666)(\mathrm{n}=16)$ \\
Pnc + HI & $11448(10017)(\mathrm{n}=15)$ & $12619(\mathrm{n}=3)$ \\
\hline
\end{tabular}

Values are mean (SD).

HI, Haemophilus influenzae; Pnc, Streptococcus pneumoniae. three hours. The plates were rinsed four times with wash buffer. The bound enzyme was detected by incubation with tetramethylbenzidine and hydrogen peroxide as substrate and quantified by means of a microplate reader (Bio-Rad, Melville, New York, USA). The results are reported as mean (SD) IL-8 concentration $(\mathrm{pg} / \mathrm{ml})$. The sensitivity of the IL-8 assay is $10 \mathrm{pg} / \mathrm{ml}$.

STATISTICAL ANALYSIS

The statistical analysis was done by using the Student's two sample $t$ test assuming unequal variances (when comparing between all the samples obtained on days 1 and 4-5) and the Wilcoxon matched paired signed rank test (when comparing between the paired samples obtained on days 1 and $4-5)$. A p value $\leqslant 0.05$ was considered significant.

\section{Results}

One hundred and one middle ear fluid samples from 81 patients were obtained by tympanocentesis on day 1 and pathogens were isolated in 79 of $101(78 \%)$ of them. Forty seven paired samples (day 1 and day 4-5) were obtained from 39 patients. These 39 patients belonged to the initial group of 81 ; for the remaining 42 patients, either a second tympanocentesis was not performed or no fluid was obtained at the second tympanocentesis.

Ninety four pathogens were isolated in 79 culture positive samples obtained on day 1: 56 Haemophilus influenzae (all untypeable), 35 Streptococcus pneumoniae, two Moraxella catarrhalis, and one Streptococcus pyogenes. Haemophilus influenzae and $S$ pneumoniae were isolated concomitantly in 15 samples. Twenty four pathogens were isolated in 21 of 47 culture positive samples obtained on day 4-5 (19 H influenzae and five $S$ pneumoniae). Haemophilus influenzae and $S$ pneumoniae were isolated concomitantly in three samples.

Among 40 of 47 initially culture positive paired samples, eradication of the initial pathogen was achieved on day $4-5$ in 22 , whereas pathogens were still present in 18 (13 H influenzae, two $S$ pneumoniae, and three concomitant $H$ influenzae and $S$ pneumoniae). Four additional paired samples were culture negative both on day 1 and day 4-5. Haemophilus influenzae was isolated on day 4-5 in three samples that were initially culture negative.

IL-8 was detected in 96 of 101 and 46 of 47 samples obtained on days 1 and $4-5$, respectively. Table 1 shows the IL- 8 concentrations found in the 96 samples taken on day 1 . Significantly higher IL-8 concentrations were found in the samples from patients with culture positive than culture negative acute otitis media. No significant differences were found in the mean IL-8 concentrations measured on day 1 in patients with acute otitis media caused by $S$ pneumoniae versus $H$ influenzae or concomitant $S$ pneumoniae and $H$ influenzae.

Table 2 shows the IL- 8 concentrations in the 47 paired samples, according to the aetiology and bacteriological outcome of acute otitis media. IL- 8 concentrations fell on day $4-5$ in 12 of 22 ears where bacterial eradication was 
Table 2 Interleukin 8 (IL-8) concentrations ( $\mathrm{pg} / \mathrm{ml}$ ) in 47 paired middle ear fluid samples, relation to aetiology of acute otitis media and day of tympanocentesis

\begin{tabular}{llc}
\hline & Day 1 & Day 4-5 \\
\hline All samples & $13517(29358)$ & $9980(11206)$ \\
Culture (+), Culture (-) $(\mathrm{n}=22)$ & $19954(41712)$ & $6695(5092)$ \\
Culture (+), Culture (+) $(\mathrm{n}=18)$ & $10048(7942)$ & $15419(15419)$ \\
HI (+), HI (-) $(\mathrm{n}=8)$ & $34486(5652)$ & $8080(5969)$ \\
HI (+), HI (+) $(\mathrm{n}=11)$ & $8412(9594)$ & $13551(17666)$ \\
Pnc (+), Pnc (-) $(\mathrm{n}=9)$ & $13517(29358)$ & $9980(11206)$ \\
\hline
\end{tabular}

Values are means $(\mathrm{SD})$

In the first column, the first set of results is for day 1 and the second set is for day $4-5$.

HI, Haemophilus influenzae; Pnc, Streptococcus pneumoniae; +, positive; -, negative.

achieved, but also in nine of 21 ears with persistent or new infection (not significant). IL-8 concentrations fell on day 4-5 compared with day 1 in the 22 samples from the patients in whom eradication was achieved. However, this decrease was not significant. Mean IL-8 concentrations did not decrease on day 4-5 compared with day 1 in the 18 samples where the pathogens persisted. IL-8 concentrations decreased on day 4-5 compared with day 1 in the samples where $S$ pneumoniae (nine samples) or $H$ influenzae (eight samples) were eradicated. However, this decrease was not significant. Mean IL-8 concentrations did not decrease on day $4-5$ compared with day 1 in the 11 samples where $H$ influenzae was not eradicated.

Mean IL-8 concentrations measured on day 4-5 were significantly higher in the 21 culture positive than in the 26 culture negative middle ear fluid samples.

\section{Discussion}

The inflammatory process associated with acute otitis media is complex and involves many factors, including bacteria, viruses, macrophages, and cytokines. ${ }^{4{ }^{12-14}}$ IL-8 is a cytokine produced mainly by monocytes, macrophages, endothelial cells, and epithelial cells. ${ }^{15}$ It acts as a chemotactic factor for neutrophils, $\mathrm{T}$ lymphocyte subsets, and basophils. In addition, it activates neutrophils to release lyposomal enzymes, undergo a respiratory burst, and degranulate and also increases the adherence of neutrophils to endothelial cells. ${ }^{16}{ }^{17}$

Our study shows the important role played by IL- 8 in the inflammatory process associated with acute otitis media and provides new information on its dynamics during antibiotic treatment. We found that IL-8 concentrations were significantly higher in bacterial acute otitis media than in culture negative acute otitis media, and no differences were found in the magnitude of the IL- 8 response to each of the two main pathogens causing the disease ( $S$ pneumoniae and $H$ influenzae). Furthermore, IL-8 concentrations were not higher in samples from those patients with acute otitis media caused by $S$ pneumoniae and $H$ influenzae concomitantly.

It is possible that increased polymorphonuclear cell migration, as inferred by higher IL-8 concentrations, is responsible for increased tissue injury and the more severe inflammatory process associated with bacterial acute otitis media. However, because viruses were shown to contribute synergistically with bacteria to the inflammatory process in acute otitis media, ${ }^{18}$ and because we did not try to isolate viruses from the middle ear fluid from our patients, the exact contribution of different viruses to the inflammatory process in this disease is yet to be established.

The high rate of spontaneous clinical recovery from acute otitis media makes it difficult to assess the contribution of antibiotic treatment to the outcome of the disease. However, there is increasing evidence that most clinical failures in the antibiotic treatment of acute otitis media are associated with bacteriological failures. ${ }^{19} 20$ Our study provides information suggesting that middle ear fluid IL-8 concentrations could potentially be used in monitoring the inflammatory process and the efficacy of different treatment modalities. Our data suggest that a decrease in IL- 8 concentrations is often found after 72-96 hours of antibiotic treatment in samples where the pathogen has been successfully eradicated, whereas IL-8 concentrations in those samples where the pathogen has not been eliminated do not decrease.

In conclusion, our study documented high concentrations of IL-8 in the middle ear fluid of children with acute otitis media before and during antibiotic treatment. We have also shown that higher IL-8 concentrations are found in the middle ear fluid from patients with culture positive acute otitis media than in those with culture negative disease. Our data showed a decrease in the magnitude of the inflammatory process, as reflected by IL-8, in the middle ear fluid of those patients where the pathogen was successfully eradicated, indirectly supporting the suggestion that early bacterial eradication is crucial to the successful outcome of acute otitis media. Further studies (including larger numbers of patients) determining the presence and role of IL- 8 and other cytokines in middle ear fluid and the correlation between immunological and bacteriological parameters with clinical outcome in acute otitis media are needed.

1 Klein JO. Otitis media. Clin Infect Dis 1994;19:823-33 2 Berman S. Otitis media in children. N Engl F Med 1995:332: 1560-5.

3 Bernstein JM. Immunologic reactivity in the middle ear in otitis media with effusion. Otolaryngol Clin North Am 1991; 24:845-59.

4 Saez-Llorens X. Pathogenesis of acute otitis media. Pediatr Infect Dis F 1994;13:1035-8.

5 Fergie JE, Purcell $\mathrm{K}$. The role of inflammatory mediators and anti-inflammatory drugs in otitis media. Pediatr Ann $1998 ; 27 ; 76-81$.

6 Yellon RF, Leonard G, Marucha PT, et al. Characterization of cytokines present in middle ear effusions. Laryngoscope 1991;101:165-9.

7 Ovesen T, Ledet T. Bacteria and endotoxin in middle ear fluid and the course of secretory otitis media. Clin fluid and the course of
Otolaryngol 1992;17:531-4.

8 Takeuchi K, Maesako K, Yuta A, Sakakura Y. Interleukin-8 gene expression in middle ear effusions. Ann Otol Rhinol Laryngol 1994;103:404-7.

9 Maxwell KS, Fitzgerald JE, Burleson JA, Leonard G, Carpenter R, Kreutzer DL. Interleukin-8 expression in otitis media. Laryngoscope 1994;104:989-95.

10 Storgaard M, Larsen K, Blegvad S, et al. Interleukin-8 and chemotactic activity of middle ear effusions. F Infect Dis 1997;175:474-7.

11 Dagan R, Abramson O, Leibovitz E, et al. Bacteriologic response to oral cephalosporins: are established susceptibility breakpoints appropriate in the case of acute otitis media? F Infect Dis 1997;176:1253-9.

12 Lewis DM, Meadema SJ, Schron JL, Lim DL. Experimental otitis media in chinchillas. Ann Otol Rhinol Laryngol 1980;89(suppl 68):344-50. 
13 Bernstein JM, Humbert JR, Hliwa MM. Oxygen consumption of middle ear and peripheral blood neutrophils in acute suppurative otitis media. Am 7 Otolaryngol 1985;6: 169-72.

14 Patel J, Chomaitree T, Schmalsteig F. Effect of modulation of polymorpholeukocyte migration with anti-CD18 antibody on pathogenesis of experimental otitis media in guinea pigs. Infect Immun 1993;61:1132-5.

15 Baggiolini M, Walz A, Kunkel SL. Neutrophil-activating peptide 1 /interleukin 8 , a novel cytokine that activates neutrophils. 7 Clin Invest 1989;84:1045-9.

16 Mukaida N, Harada A, Yasumoto K, Matsushima K. Properties of proinflammatory cell type-specific leukocyte chemotactic cytokines, interleukin-8 (IL-8) and monocyte chemotactic and activating factor (MCAF). Microbiol Immunol 1992;36:773-89.
17 Miller MD, Krangel MS. Biology and biochemistry of the chemokines: a family of chemotactic and inflammatory cytokines. Crit Rev Immunol 1992;12:17-46.

18 Chonmaitree T, Patel JA, Garofalo R, et al. Role of leukotriene B4 and interleukin-8 in acute bacterial and viral otitis media. Ann Otol Rhinol Laryngol 1996;105:96874.

19 Carlin SA, Marchant CA, Shurin PA, Johnson CE, Super DM, Rehmus JM. Host factors and early therapeutic response in acute otitis media. F Pediatr 1991;118:178-83. 20 Dagan R, Leibovitz E, Greenberg D, Yagupsky P, Fliss DM, Leiberman A. Early eradication of pathogens from middle ear fluid during antibiotic treatment of acute otitis media is associated with improved clinical outcome. Pediatr Infect Dis f 1998;17:776-82. 